\title{
Escala de habilidades de Solución de Problemas en Directivos: Desarrollo y validación
}

\author{
Scale of Problem-Solving skills in Managers: \\ Development and validation
}

\author{
Cynthia Zaira Vega-Valero * ${ }^{1}$, Rosa Alejandra Hernández-Toledano ${ }^{1}$, Oscar García-Arreola ${ }^{1}$, \\ Carlos Nava-Quiroz ${ }^{1}$, David Ruíz-Méndez ${ }^{1}$. \\ 1 - Carrera y Posgrado de Psicología, Facultad de Estudios Superiores Iztacala, Tlalnepantla, México. \\ Recibido: 13/06/19 Revisado: 01/07/19 Aceptado: 17/07/19 \\ Introducción \\ Método \\ Resultados \\ Discusión \\ Conclusiones \\ Referencias
}

\section{Resumen}

El presente estudio tuvo por objetivo el desarrollo y validación de una escala que evalúa las habilidades conductuales para resolver un problema en directivos organizacionales. Se realizó un estudio transversal ex post-facto con una muestra de 202 directivos mexicanos con al menos un año de antigüedad. Se realizó análisis de consistencia interna, análisis factorial exploratorio y confirmatorio. Se encontró una estructura tridimensional con 18 reactivos, de confiabilidad aceptable, índices de bondad de ajuste adecuados. La estructura factorial se corroboró mediante un análisis confirmatorio, con índices de ajuste absoluto aceptables. Se concluye que la escala permite contar con una medida válida y confiable de las habilidades conductuales de solución de problemas en directivos mexicanos.

Palabras clave: habilidades directivas, análisis psicométrico, solución de problemas, directivos organizacionales, análisis factorial exploratorio, análisis factorial confirmatorio

\begin{abstract}
The objective of this study was the development and validation of a scale that evaluates problem-solving behavioral skills in organizational managers. An ex post-facto cross-sectional study was carried out with a sample of 202 Mexican managers with at least a length of one year in service. An internal consistency, exploratory and confirmatory factor analyses were performed. An 18 items, three-dimensional structure was found, with acceptable reliability and adequate fit indices. The factorial structure was corroborated by a confirmatory analysis. It is concluded that the scale provides a valid and reliable measurement of problem-solving skills in Mexican managers.
\end{abstract}

Key words: management skills, psychometric analysis, problem solving, organizational management, exploratory factor analysis, confirmatory factor analysis

\footnotetext{
* Correspondencia a: Cynthia Zaira Vega Valero, Av. De los Barrios Núm. 1, Los Reyes Iztacala, 54090 Tlalnepantla, Edo. de México. Teléfono 015256231294. E-mail: vegavalero@hotmail.com

Cómo citar este artículo: Vega-Valero, C. Z., Hernández-Toledano, R. A., García-Arreola, O., Nava-Quiroz, C., \& Ruíz-Méndez, D. (2019). Escala de habilidades de solución de problemas en directivos: Desarrollo y validación. Revista Evaluar, 19(3), 68-85. Recuperado de https://revistas.unc.edu.ar/index. php/revaluar
}

Nota de autor: La construcción de este artículo fue posible gracias al financiamiento correspondiente al proyecto PAPIIT-UNAM IT300218. 


\section{Introducción}

La identificación de habilidades relevantes para el desempeño de los directivos es un tema de gran interés para la psicología organizacional (Analoui, Labbaf, \& Noorbaksh, 2000). El comportamiento directivo está compuesto por habilidades directivas que posibilitan el éxito de una persona en su función. Dichas habilidades conductuales se diferencian de atributos mentales, de la personalidad o características innatas del líder, ya que el individuo puede adquirirlas, practicarlas o mejorarlas (Sánchez, Aguirre, Barrales, Vergara, \& Mota, 2015; Whetten \& Cameron, 2011).

Son múltiples las investigaciones que han identificado aquellas habilidades que vuelven eficaces a los directivos. Entre estas, la solución de problemas es altamente valorada por las empresas, los reclutadores, los propios directivos $\mathrm{y}$ sus subordinados (American Management Association, 2012; Cardona-Puello \& Barrios-Salas, 2015; Patrón-Cortés \& Barroso-Tanoira, 2015; Patrón-Cortés, Pérez-Canul, \& Medina-Blum, 2014; Patrón-Cortés, Pérez-Canul, \& González-García, 2015; Pereda-Pérez, López-Guzmán-Guzmán, \& González-Santa-Cruz, 2014; Rodríguez, Rabazo, \& Naranjo, 2015; Whetten \& Cameron, 2011).

La investigación e intervención relativa a la solución de problemas presenta mayor consenso y volumen de estudios empíricos en la psicología clínica (Hemphill \& Littlefield, 2001; Merrill, Smith, Cumming, \& Daunic, 2017; Nezu, Nezu, \& D'Zurilla, 2014; Tenhula et al., 2014). En esta área, se cuenta con diferentes alternativas de evaluación como los inventarios de Heppner y Petersen (1982), el inventario de solución de problemas sociales de D'Zurilla y Chang (1995), la escala de resolución de problemas para adultos de Palomar-Lever, Harari-Quinn y Juárez (2010) y el inventario para evaluar a adultos coreanos de Lee, Park y Choi (2008).

Por otra parte, en el área organizacional se han reportado diferentes marcos teóricos para abordar la solución de problemas (Codina \& Rivera, 2001; Łyp-Wrońska, 2016; Pérez-Rave \& González-Echavarría, 2018). Sin embargo, la falta de consenso dificulta diferentes aspectos como la investigación, la intervención y, principalmente, la evaluación (Cohen \& Levinthal, 1990).

El área organizacional carece de herramientas para la evaluación de solución de problemas dirigidas al contexto laboral (Aguilar-Morales \& Vargas-Mendoza, 2010). Esta situación ha promovido la utilización de los inventarios antes mencionados para evaluar a los directivos en relación con la solución de problemas (Katz \& Ziderman, 1990). Una consecuencia de esta práctica es que los datos obtenidos no sirven para crear un perfil sobre las habilidades directivas y limitan mucho la información proporcionada por la medida (Ruiz, Nava, \& Carbajal, 2016).

Por otro lado, la inconsistencia teórica en los abordajes existentes es un punto crucial. Al contrastar la literatura, emergen dos áreas de oportunidad importantes: 1) muchas de las variables estudiadas bajo las condiciones previamente descritas dentro del área pertenecen a un nivel de análisis distinto del individual (Shipper \& Davy, 2002) y 2) las agrupaciones propuestas de variables presentan una amplia divergencia a nivel conceptual en los constructos psicológicos y de otras disciplinas, un caso especialmente representativo es el comportamiento directivo (Chiavenato, 2009; Robins \& Judge, 2009; San Román-Losada, 2014).

Ante esta situación, una alternativa es estudiar las habilidades de solución de problemas en el nivel individual, coherente a nivel psicológico, con el objetivo de disponer de definiciones 
precisas y empíricamente contrastables en términos conductuales, contando con indicadores potencialmente relacionables con el desempeño organizacional que presenta un individuo a un nivel empírico directo (Davis \& Luthans, 1979; Katz, 1974; Yuk1, 1989). La presente investigación propone ubicar las habilidades de solución de problemas como variables psicológicas cuyas definiciones constitutivas se relacionen parsimoniosamente con ocurrencias del comportamiento específicas, evaluables en término de su frecuencia de emisión. Para esto, se escogió el modelo teórico de Anderson et al. (2019), en el que se considera a la solución de problemas como el despliegue de conductas específicas implicadas en la resolución de un área de oportunidad organizacional. Se parte de siete habilidades que se organizan en tres grupos generales: a) estructuración del problema, que incluye: definir el problema, identificar la alternativa y determinar los criterios; b) análisis del problema, que incluye: evaluar las alternativas y elegir una; y c) resultados, que comprende: implementar la decisión tomada y evaluar los resultados obtenidos. Con base en este modelo, se elaboró una escala de medición que diera cuenta de estas siete habilidades. Un estudio previo manifestó la validez de contenido de la escala (Hernández, 2018).

El objetivo de esta investigación fue evaluar la consistencia interna y la estructura factorial de una escala que mida las habilidades conductuales en los directivos para resolver un problema en la organización.

\section{Método}

Participantes

El muestreo realizado fue no aleatorio intencional y la muestra estuvo integrada por 202 participantes voluntarios. El criterio de inclusión fue que ocuparan un puesto directivo con al menos un año de antigüedad y, por lo menos, un subordinado a su cargo. En la Tabla 1 se exhiben los porcentajes de variables según sexo, lugar de procedencia, escolaridad, experiencia gerencial previa, sector, antigüedad, giro y personas subordinadas de los directivos participantes.

Tabla 1

Datos sociodemográficos de la muestra de directivos.

\begin{tabular}{|c|c|}
\hline Variable & Porcentaje \\
\hline \multicolumn{2}{|l|}{ Sexo } \\
\hline Hombre & 47.5 \\
\hline Mujer & 52.5 \\
\hline \multicolumn{2}{|c|}{ Lugar de procedencia } \\
\hline Ciudad de México & 53.5 \\
\hline Estado de México & 26.7 \\
\hline Jalisco & 4 \\
\hline Oaxaca & 2.5 \\
\hline Otros estados de la República & 13.3 \\
\hline \multicolumn{2}{|c|}{ Escolaridad } \\
\hline Primaria & 2 \\
\hline Secundaria & 5.4 \\
\hline Bachillerato & 7.9 \\
\hline Carrera técnica & 5.9 \\
\hline Licenciatura o ingeniería & 48.5 \\
\hline Posgrado & 30.2 \\
\hline \multicolumn{2}{|c|}{ Experiencia gerencial previa } \\
\hline Sí & 56.4 \\
\hline No & 43.6 \\
\hline \multicolumn{2}{|c|}{ Sector } \\
\hline Público & 44.1 \\
\hline Privado & 55.9 \\
\hline \multicolumn{2}{|c|}{ Antigüedad } \\
\hline 1 año & 16.3 \\
\hline De 2 a 3 & 30.2 \\
\hline De 4 a 7 & 27.7 \\
\hline 8 o más & 25.7 \\
\hline \multicolumn{2}{|l|}{ Giro } \\
\hline Industrial & 13.4 \\
\hline Comercio & 20.3 \\
\hline Servicios & 66.3 \\
\hline
\end{tabular}

Personas subordinadas 


\begin{tabular}{cc} 
De 1 a 3 & 20.8 \\
De 4 a 8 & 27.2 \\
De 9 a 18 & 27.2 \\
Más de 20 & 24.8 \\
\hline
\end{tabular}

Diseño

El estudio tiene un diseño transversal ex post facto, ya que los datos se recolectaron en un solo momento (Hernández-Sampieri, Fernández-Collado, \& Baptista-Lucio, 2014).

\section{Instrumento}

Para construir el instrumento Escala de Solución de Problemas en Directivos (ESOP-D), se tomó como referencia el modelo de Anderson et al. (2019) para la elaboración y organización de los ítems. La ESOP-D quedó compuesta por 40 ítems tipo Likert, con cinco opciones de respuesta en términos de frecuencia: (1) Nunca, (2) Casi nunca, (3) Algunas veces, (4) Casi siempre, (5) Siempre. Cada habilidad fue medida a partir de las siguientes estructuras: habilidad para definir el problema (seis ítems: 6, 11, 16, 18, 19, 36), habilidad para identificar alternativas (seis ítems: 2, 10, 13, 17, 31, 33 ), habilidad para determinar los criterios de solución (cinco ítems: 9, 12, 14, 34, 35), habilidad para evaluar alternativas de solución (siete ítems: 4, 20, 22, 26, 29, 38, 39), habilidad para elegir una alternativa (cuatro ítems: 5, 23, 24, 40), habilidad de implementación de la solución (seis ítems: 3, 8, 15, 21, 30, 32), habilidad de evaluación de resultados (seis ítems: 1, 7, 25, 27, 28, 37). Posteriormente, los ítems fueron sometidos al criterio de seis jueces expertos en la construcción de instrumentos y en la evaluación de habilidades en contextos organizacionales para evaluar la relevancia y validez del contenido en cada habilidad con base a los criterios establecidos por Escobar-Pérez y Cuervo-Martínez (2008) entre los cuales se encuentran la experiencia en la realización de juicios y toma de decisiones basada en evidencia, el grado académico, la realización de investigaciones en el campo, el estatus en la comunidad, las publicaciones en el campo y los reconocimientos recibidos. Las sugerencias realizadas permitieron desechar o reformular varios reactivos para adaptarlos al ámbito organizacional. El orden de la presentación de los ítems fue asignado de manera aleatoria (ver Apéndice 1).

\section{Procedimiento}

La aplicación de la ESOP-D se realizó de dos maneras distintas. En la primera se invitó a los directivos a contestar la escala por vía electrónica en la plataforma Typeform. En la segunda se visitaron las instalaciones de distintas organizaciones para realizar la aplicación presencial con la escala impresa. En ambos casos, se les brindó a los participantes un consentimiento informado para autorizar el uso de la información proporcionada, en el que se garantizaba la confidencialidad de sus datos y se aseguraba que la medición no suponía una evaluación de desempeño. La aplicación de la escala en estas dos instancias a la muestra presentada tuvo una duración de dos meses. La resolución de la escala requiere 20 minutos aproximadamente.

\section{Análisis de datos}

Para el procesamiento estadístico se utilizaron los programas SPSS versión 22 (IMB, 2013) AMOS versión 22 (IBM, 2013) y Factor versión 10.10.01 (Lorenzo-Seva \& Ferrando, 2019). La confiabilidad del instrumento se evaluó a partir 
de la consistencia interna de los ítems empleando el coeficiente alfa de Cronbach. Se eliminaron los ítems que presentaran correlaciones ítem total inferiores a .20, como lo sugieren Cozby (2005) y Quero-Virla (2010). Para el análisis factorial exploratorio (AFE), se utilizaron los criterios de Lloret-Segura, Ferreres-Traver, Hernández-Baeza y Tomás-Marco (2014). Se utilizaron criterios numéricos y teóricos para identificar estructuras factoriales probables, considerando como criterios aceptables para factorizar: el índice Kaiser-Mayer-Olkin (KMO) superior a .80, la prueba de esfericidad de Barlett significativa y el determinante superior a cero. En el AFE se aplicaron los siguientes criterios: cargas factoriales superiores a .40 , que estas discriminaran al menos en .10 con respecto a las cargas secundarias del ítem, que cada dimensión esté constituida por, al menos, tres ítems, y que la agrupación de los ítems en los factores tenga congruencia teórica.

Se realizó un análisis factorial confirmatorio (AFC) con cada uno de los modelos posibles y se contrastaron con base en indicadores de ajuste y parsimonia como indican Lévy-Mangin, Martín-Fuentes y Román-González (2006). Se evaluó la distribución empírica de la muestra con la prueba Shapiro-Wilk, como recomiendan Razali y Wah (2011). Además, se realizaron pruebas de comparación de medias entre variables sociodemográficas consideradas relevantes en la literatura, para corroborar si la escala es sensible para detectar diferencias en cuestión de grados de la habilidad.

\section{Hipotesis}

De acuerdo con Echavarri, Godoy y Olaz (2007), las estrategias utilizadas para solucionar problemas complejos entre hombres y mujeres son diferentes, el razonamiento espacial y abstracto es mayormente empleado por varones, mientras que las mujeres presentan un mayor uso de estrategias verbales. Sin embargo, no se establece cuál de estas estrategias sería la más efectiva, por lo que se espera que no se encuentren diferencias entre hombres y mujeres en las habilidades de solución de problemas.

No se lograron identificar investigaciones previas que realicen contraste entre el sector $\mathrm{y}$ giro de la empresa donde trabaja el directivo. No obstante, se espera un mayor grado de habilidad en aquellos directivos provenientes del sector privado y del giro de servicios debido a que este sector se caracteriza por el desarrollo y fortalecimiento de habilidades que pueden ser un factor diferencial en mercados de alta competitividad. Por su parte, el giro de servicios se define por una gran variabilidad de problemas, ya que los servicios se ajustan constantemente a las necesidades de los consumidores.

Mumford, Marks, Zaccaro y Raiter-Palmon (2000) señalan que la habilidad para solucionar problemas se desarrolla a lo largo de la carrera del directivo, por lo que se espera que aquellos que tengan mayor antigüedad dentro de una empresa presenten un mayor grado de esta habilidad. Lo mismo se supone con respecto a la escolaridad, aquel directivo que cuente un mayor nivel educativo tendrá mayor grado de habilidad de solución de problemas (Pereda-Pérez et al., 2014).

Respecto del número de subordinados que le reportan al directivo, en la literatura se ha indicado que la eficacia de los equipos, así como la coordinación de estos para solucionar un problema en la organización, depende del número de sus integrantes, a mayor número de integrantes aumenta su eficacia hasta llegar a un punto de decremento en la eficiencia de los recursos humanos con los que cuenta el directivo (Rangel-Carreño, Lugo-Garzón, \& Calderón, 2018; Rico, Alcover de la Hera, \& Tabernero, 2010). Por tal razón, se 
espera encontrar mayor ejercicio de habilidades en directivos que manejen un número moderado de subordinados, en comparación con aquellos directivos que cuenten con un número limitado de trabajadores, así como con un número alto de los mismos.

\section{Resultados}

El primer paso para analizar los datos fue evaluar la consistencia interna del instrumento. En este paso se eliminó el ítem 31 por su correlación ítem-total menor a 20 y así se obtuvo un alfa de .95. Este valor puede ser visto como un posible indicio de redundancia (Celina-Oviedo \& Campo-Arias, 2005). Sin embargo, se decidió mantener los ítems restantes para analizar las posibles estructuras en el AFE (ver Tabla 2).

Una vez que se obtuvo la consistencia interna, se procedió a realizar el AFE utilizando el método de extracción por máxima verosimilitud y rotación varimax, debido a que teóricamente las habilidades son independientes. Los índices KMO (.93), la prueba de esfericidad de Barlett $\left(\chi^{2}\right.$ $(741)=4755.99, p<.001)$ y el determinante $(9.21$ e-9) indican que es factible realizar

los análisis.

La identificación del número de dimensiones posibles se realizó con base en criterios teóricos y posteriormente de tipo numérico. Para el primer modelo se realizó el AFE eligiendo siete factores de acuerdo con el modelo propuesto por Anderson et al. (2019). Se identificó que algunos factores no contaban con al menos tres ítems por dimensión, por lo que se optó por los tres grupos teóricos generales que agrupan dichas habilidades citadas en la introducción, como se muestra en la Tabla 3. Los ítems se agrupan de acuerdo con esta estructura por lo que la primera dimensión se nombró estructuración del problema (ocho ítems), la segunda análisis del problema (siete ítems) y la tercera implementación y evaluación de los resultados (tres ítems). Por otro lado, con base en criterios numéricos, se establecieron dos dimensiones a partir de la prueba de análisis paralelo (AP; Timmerman \& Lorenzo-Seva, 2011); sin embargo, este modelo no cumple con el criterio de coherencia teórica ya que agrupa ítems de manera indistinta.

Tabla 3a

Análisis factorial exploratorio del modelo con tres dimensiones.

\begin{tabular}{|c|c|c|c|}
\hline \multicolumn{4}{|c|}{ Modelo 1} \\
\hline \multirow[t]{2}{*}{ Ítems } & \multicolumn{3}{|c|}{ Factor } \\
\hline & 1 & 2 & 3 \\
\hline 6 & .74 & .20 & .20 \\
\hline 5 & .70 & .16 & .17 \\
\hline 4 & .65 & .12 & .25 \\
\hline 16 & .59 & .30 & .35 \\
\hline 17 & .58 & .34 & .13 \\
\hline 11 & .55 & .26 & .24 \\
\hline 12 & .53 & .36 & .15 \\
\hline 2 & .51 & .25 & .01 \\
\hline 33 & .23 & .66 & .20 \\
\hline 39 & .29 & .65 & .27 \\
\hline 22 & .24 & .63 & .27 \\
\hline 23 & .33 & .61 & .34 \\
\hline 26 & .33 & .58 & .35 \\
\hline 20 & .25 & .57 & .43 \\
\hline 24 & .38 & .51 & .37 \\
\hline 35 & .19 & .34 & .77 \\
\hline 34 & .25 & .31 & .73 \\
\hline 30 & .21 & .38 & .57 \\
\hline Alfa & .82 & .84 & .83 \\
\hline
\end{tabular}

Tabla 3b

Análisis factorial exploratorio del modelo con dos dimen- 
siones.

\begin{tabular}{|c|c|c|}
\hline \multicolumn{3}{|c|}{ Modelo } \\
\hline \multirow[t]{2}{*}{ Items } & \multicolumn{2}{|c|}{ Factor } \\
\hline & 1 & 2 \\
\hline 35 & .71 & .23 \\
\hline 34 & .70 & .28 \\
\hline 26 & .69 & .36 \\
\hline 29 & .69 & .13 \\
\hline 30 & .68 & .24 \\
\hline 39 & .66 & .32 \\
\hline 27 & .65 & .31 \\
\hline 19 & .65 & .32 \\
\hline 23 & .64 & .38 \\
\hline 20 & .64 & .31 \\
\hline 28 & .63 & .34 \\
\hline 25 & .62 & .36 \\
\hline 21 & .61 & .37 \\
\hline 24 & .61 & .42 \\
\hline 33 & .61 & .26 \\
\hline 22 & .59 & .30 \\
\hline 13 & .57 & .30 \\
\hline 32 & .49 & .16 \\
\hline 6 & .24 & .76 \\
\hline 15 & .21 & .71 \\
\hline 5 & .19 & .68 \\
\hline 17 & .29 & .64 \\
\hline 4 & .25 & .62 \\
\hline 16 & .44 & .62 \\
\hline 11 & .30 & .59 \\
\hline 7 & .37 & .59 \\
\hline 12 & .33 & .57 \\
\hline 2 & .17 & .51 \\
\hline 14 & .36 & .51 \\
\hline 8 & .29 & .43 \\
\hline Alfa & .94 & .89 \\
\hline
\end{tabular}

También se consideró la posibilidad de una estructura unidimensional en la escala, por lo que se obtuvieron coeficientes de consistencia interna de división de mitades de Guttman $=.92$ y Spearman-Brown $=.93$. Se observó: 1) la varianza explicada para el primer factor cercana al $40 \%$ (39.66\% de la varianza), y 2) el decaimiento entre el primer factor y el segundo con el gráfico de sedimentación (ver Figura 1).

Para tomar la decisión de cuál es la mejor estructura factorial, se realizó el AFC de cada uno de los modelos para compararlos a partir de sus indicadores de bondad de ajuste. Como se observa en la Tabla 3, el CFI, GFI, AGFI y NNFI muestran valores superiores en el modelo de tres dimensiones con respecto a los otros dos, indicando menor error, mejor ajuste y mayor coherencia teórica (ver Tabla 4).

En síntesis, la escala total queda compuesta por 18 ítems que explican el 61.74\% de la varianza acumulada y una consistencia interna total de $\alpha$ $=.93$. El primer factor nombrado estructuración del problema se compone de ocho ítems $(6,5,4$, $16,17,11,12,2)$ que corresponden a las habilidades de definir el problema, identificar alternativas y determinar criterios, que presentan una varianza explicada del $47.55 \%$ con un alfa de .87 . El segundo factor, nombrado análisis de alternativas del problema se compone de siete reactivos (33, $39,22,23,26,20,24)$ de las habilidades: evaluar alternativas y elegir una alternativa, las cuales presentan el $9.12 \%$ de la varianza explicada y un coeficiente de confiabilidad de .89. El tercer factor, implementación y evaluación de los resultados, está conformado por tres ítems $(35,34$, 30) que corresponden a las habilidades de implementar la decisión y evaluar los resultados, que presenta $5.46 \%$ de la varianza explicada con un alfa de .83. Lo anterior implica que la escala cumple con los criterios de confiabilidad y validez de Cozby (2005) y Lloret-Segura et al. (2014).

Una vez que se establecieron tres factores se procedió a identificar si los datos se distribuyen normalmente de acuerdo con las dimensiones a través de la prueba Shapiro-Wilk como lo indican Razali y Wah (2011). Los resultados mues- 
Tabla 2

Análisis de confiabilidad general.

\begin{tabular}{lccccccccc}
\hline Ítem & $\overline{\mathbf{X}}$ & $\mathbf{D E}$ & $\boldsymbol{r}$ & $\begin{array}{c}\boldsymbol{\alpha} \text { sin el } \\
\text { elemento }\end{array}$ & $\mathbf{\text { Ítem }}$ & $\overline{\mathbf{X}}$ & $\mathbf{D E}$ & $\boldsymbol{r}$ & $\begin{array}{c}\boldsymbol{\alpha} \text { sin el } \\
\text { elemento }\end{array}$ \\
\hline 1 & 3.74 & 1.08 & .23 & .95 & 21 & 4.29 & 0.82 & .67 & .95 \\
2 & 4.27 & 0.78 & .46 & .95 & 22 & 4.10 & 0.87 & .61 & .95 \\
3 & 3.35 & 1.25 & .56 & .95 & 23 & 4.15 & 0.89 & .71 & .95 \\
4 & 4.11 & 0.91 & .58 & .95 & 24 & 4.34 & 0.68 & .71 & .95 \\
5 & 4.30 & 0.81 & .56 & .95 & 25 & 4.20 & 0.85 & .70 & .95 \\
6 & 4.33 & 0.81 & .65 & .95 & 26 & 4.22 & 0.86 & .73 & .95 \\
7 & 4.24 & 0.88 & .66 & .95 & 27 & 4.18 & 0.90 & .68 & .95 \\
8 & 4.09 & 0.87 & .52 & .95 & 28 & 4.17 & 0.95 & .70 & .95 \\
9 & 4.05 & 0.97 & .53 & .95 & 29 & 3.58 & 1.14 & .60 & .95 \\
10 & 4.12 & 0.83 & .66 & .95 & 30 & 4.15 & 0.93 & .66 & .95 \\
11 & 4.09 & 0.93 & .59 & .95 & 31 & 2.67 & 1.14 & .12 & .96 \\
12 & 4.43 & 0.74 & .58 & .95 & 32 & 4.17 & 0.94 & .47 & .96 \\
13 & 4.15 & 0.84 & .61 & .95 & 33 & 4.23 & 0.85 & .61 & .95 \\
14 & 4.62 & 0.61 & .58 & .95 & 34 & 4.17 & 0.92 & .70 & .95 \\
15 & 4.30 & 0.73 & .61 & .95 & 35 & 3.77 & 1.14 & .68 & .95 \\
16 & 4.30 & 0.79 & .71 & .95 & 36 & 4.20 & 0.89 & .62 & .95 \\
17 & 4.48 & 0.68 & .63 & .95 & 37 & 4.41 & 0.83 & .53 & .95 \\
18 & 4.33 & 0.77 & .66 & .95 & 38 & 4.21 & 0.95 & .50 & .96 \\
19 & 4.27 & 0.88 & .68 & .95 & 39 & 4.18 & 0.90 & .70 & .95 \\
20 & 4.14 & 0.91 & .65 & .95 & 40 & 4.31 & 0.82 & .30 & .96
\end{tabular}

Nota: $\overline{\mathrm{X}}$ media muestral, $\mathrm{DE}=$ desviación estándar, $r=$ correlación ítem total.

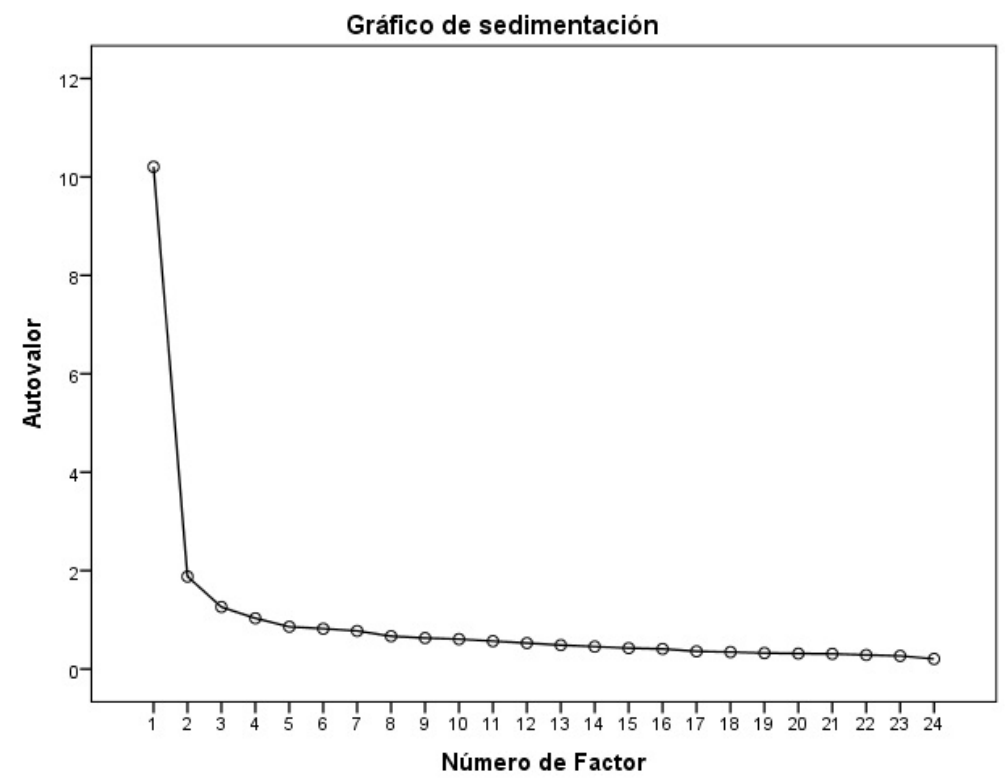


Tabla 4

Comparación de índices de bondad de ajuste de las posibles dimensiones.

\begin{tabular}{|c|c|c|c|c|c|c|}
\hline \multicolumn{7}{|c|}{ Índices de bondad de ajuste } \\
\hline Modelo & $\begin{array}{c}\chi^{2}(g l) \\
p\end{array}$ & CFI & GFI & AGFI & NNFI & RMSEA \\
\hline Una dimensión & $\begin{aligned} \chi_{(702)}^{2} & =1700.96 \\
p & <.001\end{aligned}$ & .77 & .66 & .62 & .67 & .08 \\
\hline Dos dimensiones & $\begin{array}{c}\chi_{(404)}^{2}=912.95 \\
p<.001\end{array}$ & .85 & .77 & .73 & .77 & .08 \\
\hline Tres dimensiones & $\begin{array}{c}\chi_{(132)}^{2}=240.36 \\
p<.001\end{array}$ & .94 & .89 & .85 & .88 & .06 \\
\hline
\end{tabular}

Nota. Razón Chi-cuadrado/ grados de libertad $\left(\chi^{2} / \mathrm{gl}\right)$ : Se recomiendan valores mayores que 2. Índice de ajuste Comparativo (CFI), Índice de bondad de ajuste (GFI), Índice de bondad de ajuste ajustado (AGFI), Índice de ajuste No Normalizado (NNFI), Raíz cuadrada del error medio cuadrático (RMSEA).

Tabla 5

Prueba de normalidad de las dimensiones de la escala.

\begin{tabular}{lccc}
\hline & \multicolumn{3}{c}{ Shapiro-Wilk } \\
\hline & Estadístico & $\boldsymbol{g l}$ & Sig. \\
\hline Puntaje total del ESOP-D & .95 & 202 & $<.001$ \\
Factor Habilidad de estructuración del problema & .92 & 202 & $<.001$ \\
Factor Habilidad de análisis de alternativas del problema & .92 & 202 & $<.001$ \\
Factor Habilidad de implementación y evaluación de resultados & .90 & 202 & $<.001$
\end{tabular}

Tabla 6

Prueba de normalidad de las dimensiones de la escala.

\begin{tabular}{|c|c|c|c|c|c|c|c|c|c|c|}
\hline Variables & $\mathrm{X} 1$ & DE & $\mathrm{X} 2$ & DE & $\mathbf{X 3}$ & DE & $\mathrm{X4}$ & $\mathbf{D E}$ & $\begin{array}{r}\text { Diferencia de } \\
\text { medias }\end{array}$ & $\begin{array}{r}\text { Tamaño del } \\
\text { efecto }\end{array}$ \\
\hline Sexo & \multicolumn{2}{|c|}{ Hombres } & \multicolumn{2}{|c|}{ Mujeres } & & & & & & \\
\hline Puntaje total & 75.08 & 10.35 & 76.37 & 10.77 & & & & & $\begin{array}{c}t=-.869(200) \\
p=.386\end{array}$ & $d=-.12$ \\
\hline Estructuración & 33.91 & 5.01 & 34.66 & 4.41 & & & & & $\begin{array}{c}t=-1.13(200) \\
p=.267\end{array}$ & $d=-.15$ \\
\hline Alternativas & 29.31 & 4.27 & 29.40 & 5.04 & & & & & $\begin{array}{c}t=-.141(200) \\
p=.878\end{array}$ & $d=-.01$ \\
\hline Implementación & 11.85 & 2.7 & 12.30 & 2.5 & & & & & $\begin{array}{c}t=1.21(200) \\
p=.223\end{array}$ & $d=-.17$ \\
\hline
\end{tabular}

Sector Público Privado




\begin{tabular}{|c|c|c|c|c|c|c|}
\hline Puntaje total & 75.86 & 10.72 & 75.68 & 10.49 & $\begin{array}{c}t=.122(200) \\
p=.916\end{array}$ & $d=-.01$ \\
\hline Estructuración & 34.17 & 4.53 & 34.41 & 4.86 & $\begin{array}{c}t=-.353(200) \\
p=.727\end{array}$ & $d=.05$ \\
\hline Alternativas & 29.32 & 4.92 & 29.38 & 4.5 & $\begin{array}{c}t=-.096(200) \\
p=.923\end{array}$ & $d=-.01$ \\
\hline Implementación & 12.35 & 2.26 & 11.87 & 2.86 & $\begin{array}{c}t=1.304(200) \\
p=.189\end{array}$ & $d=.18$ \\
\hline
\end{tabular}

\begin{tabular}{|c|c|c|c|c|c|c|c|c|}
\hline Escolaridad & \multicolumn{2}{|c|}{ Básico } & \multicolumn{2}{|c|}{ Profesional } & \multicolumn{2}{|c|}{ Posgrado } & \multirow[b]{2}{*}{$\begin{array}{c}f=4.16(2,199) \\
p=.021\end{array}$} & \multirow[b]{2}{*}{$\eta^{2}=.04$} \\
\hline Puntaje total & 72.83 & 10.47 & 77.85 & 9.58 & 74.45 & 11.56 & & \\
\hline Estructuración & 32.32 & 5.43 & 35.57 & 3.85 & 33.68 & 4.89 & $\begin{array}{c}f=8.42(2,199) \\
p=.001\end{array}$ & $\eta^{2}=.078$ \\
\hline Alternativas & 28.69 & 4.5 & 30.04 & 4.3 & 28.73 & 5.26 & $\begin{array}{c}f=2.02(2,199) \\
p=.135\end{array}$ & $\eta^{2}=.019$ \\
\hline Implementación & 11.81 & 2.86 & 12.24 & 2.68 & 12.03 & 2.35 & $\begin{array}{c}f=.422(2,199) \\
p=.656\end{array}$ & $\eta^{2}=.004$ \\
\hline Giro & \multicolumn{2}{|c|}{ Industrial } & \multicolumn{2}{|c|}{ Comercio } & \multicolumn{2}{|c|}{ Servicios } & & \\
\hline Puntaje total & 71.29 & 10.66 & 76.65 & 8.57 & 76.38 & 10.94 & $\begin{array}{c}f=2.84(2,199) \\
p=.048\end{array}$ & $\eta^{2}=.027$ \\
\hline Estructuración & 32.74 & 4.47 & 34.68 & 3.85 & 34.51 & 4.91 & $\begin{array}{c}f=1.76(2,199) \\
p=.144\end{array}$ & $\eta^{2}=.017$ \\
\hline Alternativas & 27.37 & 5.02 & 30.02 & 3.59 & 29.55 & 4.82 & $\begin{array}{c}f=3.03(2,199) \\
p=.044\end{array}$ & $\eta^{2}=.029$ \\
\hline Implementación & 11.18 & 3.31 & 11.95 & 2.61 & 12.31 & 2.43 & $\begin{array}{c}f=2.179(2,199) \\
p=183\end{array}$ & $\eta^{2}=.021$ \\
\hline
\end{tabular}

Antigüedad 1 año 2 y 3 años 4 a 7 años 8 años o más

$\begin{array}{lllllllllll}\text { Puntaje total } & 77.21 & 7.69 & 74.11 & 11.56 & 74.82 & 9.94 & 77.78 & 11.37 & \begin{array}{c}f= \\ = \\ p=.49\end{array}(3,198) & \eta^{2}=.022\end{array}$

Estructuración $\quad 35.06 \quad 3.73 \quad 33.80 \quad 4.88 \quad 34.21 \quad 4.35 \quad 34.53 \quad 5.43 \quad f=.558(3,198) \quad \eta^{2}=.008$ 


\begin{tabular}{|c|c|c|c|c|c|c|c|c|c|c|}
\hline Alternativas & 29.48 & 3.34 & 28.83 & 5.2 & 28.51 & 4.68 & 30.80 & 4.52 & $\begin{array}{c}f=2.58(3,198) \\
p=.041\end{array}$ & $\eta^{2}=.037$ \\
\hline Implementación & 12.66 & 1.57 & 11.47 & 2.96 & 12.08 & 2.32 & 12.44 & 2.91 & $\begin{array}{c}f=1.99(3,198) \\
p=.90\end{array}$ & $\eta^{2}=.029$ \\
\hline $\begin{array}{c}\text { Número de personas } \\
\text { a su cargo }\end{array}$ & \multicolumn{2}{|c|}{ 1-3 personas } & \multicolumn{2}{|c|}{ 4-8 personas } & \multicolumn{2}{|c|}{$\begin{array}{c}\text { 9-18 perso- } \\
\text { nas }\end{array}$} & \multicolumn{2}{|c|}{$\begin{array}{c}\text { 20-75 perso- } \\
\text { nas }\end{array}$} & & \\
\hline Puntaje total & 76.23 & 1.69 & 73.63 & 11.74 & 77.41 & 9.99 & 75.88 & 9.31 & $\begin{array}{c}f=1.227(3,198) \\
p=.301\end{array}$ & $\eta^{2}=.018$ \\
\hline Estructuración & 34.73 & 4.62 & 33.90 & 4.78 & 34.34 & 4.93 & 34.36 & 4.56 & $\begin{array}{c}f=.248(3,198) \\
p=.862\end{array}$ & $\eta^{2}=.003$ \\
\hline Alternativas & 29.5 & 4.58 & 28.29 & 5.53 & 30.25 & 4.15 & 19.44 & 4.15 & $\begin{array}{c}f=1.65(3,198) \\
p=.174\end{array}$ & $\eta^{2}=.024$ \\
\hline Implementación & 12 & 2.63 & 11.43 & 3.2 & 12.81 & 2.33 & 12.08 & 1.98 & $\begin{array}{c}f=2.63(3,198) \\
p=.05\end{array}$ & $\eta^{2}=.038$ \\
\hline
\end{tabular}

Nota. ${ }^{*}$ Los intervalos de confianza de la diferencia de medias no cruzan por cero. Se utilizó el método Montecarlo y los resultados se basan en distribuciones muestrales tipo Bootstraping con 2000 muestras.

tran que los datos de ningún factor se distribuyen normalmente, por lo que se recomienda que los resultados obtenidos al utilizar pruebas paramétricas sean tratados con mesura (ver Tabla 5).

Para identificar posibles diferencias en la solución de problemas con respecto a las variables de escolaridad, sexo, edad, antigüedad, giro y sector se aplicaron pruebas de contraste de medias. Los resultados muestran diferencias en escolaridad, antigüedad, giro y sector (ver Tabla 6).

Con respecto a las diferencias encontradas en las pruebas en relación con los ANOVA, la prueba post hoc de Bonferroni indica diferencias en la escolaridad, giro, antigüedad y número de subordinados. La primera de ellas se observa en el puntaje total de la escala de acuerdo con los niveles básico y licenciatura CI 95\% Bootstraping Bca [-8.72 - -1.55] con efecto moderado $\left(\eta^{2}=\right.$
.04); la habilidad de estructuración del problema presenta diferencias respecto al nivel básico, licenciatura CI 95\% Bootstraping Bca [1.23 - 5.25] y licenciatura con posgrado CI 95\% Bootstraping Bca [.091 - 3.67] con efecto moderado ( $\eta 2$ $=.078)$.

Respecto al giro, existen diferencias en el puntaje total de la escala entre el área industrial y servicios CI 95\% Bootstraping Bca [-9.63 -.53] con un efecto significativo débil $(\eta 2=.027)$; y respecto a la habilidad de alternativas entre el giro industrial y servicios CI 95\% Bootstraping BcA [-4.42 — -.14] con un efecto significativo débil $\left(\eta^{2}=.029\right)$; sin embargo, la prueba post hoc de Bonferroni no muestra diferencias estadísticamente significativas entre los grupos por intervalos de confianza que cruzan por cero.

De acuerdo con la antigüedad reportada por 
los directivos, se presentaron diferencias para generar alternativas si tienen de cuatro a siete años y ocho o más años CI 95\% Bootstraping Bca [-3.94 - -.59] con un efecto significativo débil $\left(\eta^{2}=.022\right)$.

Finalmente, se encontraron diferencias en la habilidad de implementación en los casos con 4 a 8 subordinados y de 9 a 18 personas a su cargo CI Bootstraping Bca [-2.44 - -.37] con un efecto significativo débil $\left(\eta^{2}=.038\right)$.

\section{Discusión}

Se planteó como objetivo el desarrollo y la validación de una escala que evaluara las habilidades conductuales para resolver un problema en directivos organizacionales. Mediante los resultados se evidencia que la consistencia interna y la agrupación de los ítems en tres factores cuenta con características métricas idóneas. Asimismo, la escala se ajusta al modelo teórico propuesto por Anderson et al. (2019).

Cabe mencionar que Celina-Oviedo y Campo-Arias (2005) indican que el valor máximo esperado de alfa de Cronbach sea de .90. Nuestro caso dio como resultado un alfa general mayor a este valor; si bien esto indica redundancia o duplicación dentro del instrumento, los análisis factoriales confirmatorios, particularmente el del modelo unidimensional, presentaron los niveles más bajos de ajuste. En el caso de los niveles de confiabilidad de cada factor, estos resultaron adecuados, por tanto, los alfas solo cambiarán de acuerdo con la muestra a la que se le aplique la escala esperando que esos cambios no sean drásticos y atenten contra la consistencia de los ítems (Streiner, 2003).

Respecto de la validez se eligieron los análisis factoriales necesarios para mostrar el mejor ajuste en términos de las agrupaciones de los ítems, estos a su vez respondieron al modelo teórico de Anderson et al. (2019), lo cual permite que las puntuaciones derivadas de la escala sean interpretadas a la luz de un contexto organizacional y de la presencia o ausencia de habilidades de solución de problemas de un directivo.

Sumando a lo anterior, los datos permiten identificar diferencias respecto a las variables de identificación. En el caso de la escolaridad se distinguen diferencias a favor del nivel de licenciatura para la habilidad estructuración del problema, así como en el puntaje total de la escala. Esta condición confirma lo establecido por otros estudios en donde se destaca la formación universitaria de directivos respecto a la percepción de sus habilidades (Pereda-Pérez et al., 2014). Esto puede deberse a que en los últimos años se ha incluido a la solución de problemas en la lista de competencias que se fomentan en la formación universitaria (González, Wagenaar, \& Beneitone, 2004; Rodríguez et al., 2015) por lo que nivel de escolaridad sí influye en el desarrollo de habilidades a partir del pensamiento crítico para evaluar y elegir las alternativas en la solución de un problema (Núñez-López, Ávila-Palet, \& Olivares-Olivares, 2016).

Considerando la antigüedad también se reconocen diferencias de acuerdo con la habilidad de análisis de alternativas del problema. Los participantes que tienen más de ocho años en su puesto reportan un promedio más alto comparado a los que tienen de 4 a 7 años en su puesto de trabajo. Esta diferencia significativa se ajusta a la literatura que clasifica como "expertos" a aquellos directivos que tienen más antigüedad en la organización y "novatos" a los que recién ingresan (Gross-Salvat, 1990; Mumford, Zaccaro, Harding, Jacobs, \& Fleishman, 2000). De acuerdo con Gross-Salvat (1990) la solución suele darse de forma más satisfactoria cuando el problema es resuelto por un experto que por un novato, y la 
diferencia puede deberse a que los primeros hacen un mejor uso de las habilidades, como es el caso de la habilidad de identificar las alternativas y la habilidad para determinar los criterios de evaluación de alternativas comparado el ejercicio de habilidades de los segundos.

También se hallaron diferencias en la habilidad de implementación y evaluación de los resultados, ya que presentaron una media significativamente superior aquellos directivos que tienen de 4 a 8 subordinados, comparados con aquellos que tienen de 9 a 18 subordinados. De acuerdo con Huber (1992) al gestionar una posible solución que involucra coordinar el trabajo de sus subordinados, la efectividad potencial del grupo se determina por cuán bien se realicen las funciones de planificar, organizar y dotar del número de personas que requiere el directivo para implementar la solución de problemas, por lo que al incrementar el número de personas que le reportan directamente precisa de un mayor ejercicio de las habilidades necesarias para implementar el plan de acción.

\section{Conclusiones}

Las hipótesis planteadas sobre las diferencias en la solución de problemas en relación con las variables demográficas seleccionadas en este estudio se confirman, con excepción de la diferencia en el sector, lo que permite sugerir estudios que investiguen si las habilidades de solución de problemas en directivos del sector público y el privado son distintas. La escala de evaluación de habilidades para la solución de problemas dirigida a directivos que se presenta es una propuesta tecnológica para las organizaciones, ya que permite realizar un diagnóstico del ejercicio en los directivos, con el fin de realizar programas de capacitación que tengan como objetivo entrenar es- tas habilidades según las necesidades detectadas.

Además, hay que señalar que la escala no es una evaluación de desempeño, sino que es un primer acercamiento para identificar las habilidades de solución de problemas de los directivos con una escala desarrollada específicamente para el ámbito organizacional. Se recomienda que, después de ser aplicada, se desarrollen programas de capacitación que se enfoquen en habilidades conductuales, como lo plantea el modelo de capacitación basado en evidencias de Ruíz-Méndez y Vega-Valero (2017).

\section{Referencias}

Aguilar-Morales, J. E., \& Vargas-Mendoza, J. E. (2010). La enseñanza de la psicología aplicada a los escenarios organizacionales en México: Análisis de los contenidos curriculares. Boletín Electrónico de Investigación de la Asociación Oaxaqueña de Psicología A.C., 6(1), 57-88. Recuperado de http://www. conductitlan.net

American Management Association. (2012). Critical Skills Survey. Recuperado de https://playbook.amanet.org Analoui, F., Labbaf, H., \& Noorbakhsh, F. (2000). Identification of clusters of managerial skills for increased effectiveness: The case of the steel industry in Iran. International Journal of Training and Development, 4(3), 217-234. doi: 10.1111/1468-2419.00109

Anderson, D., Sweeney, D., Williams, T., Camm, J., Cochran, J., Fry, M., \& Ohlmann, J. (2019). Fundamentos de métodos cuantitativos para los negocios. México, D. F.: Cengage Learning.

Cardona-Puello, S. P., \& Barrios-Salas, J. S. (2015). Aprendizaje basado en problemas (ABP): El "problema" como parte de la solución. Revista Institucional Adelante Ahead, 6(3), 81-90. Recuperado de http://www. unicolombo.edu.co/ojs/index.php/adelante-ahead

Celina-Oviedo, H., \& Campo-Arias, A. (2005). Aproxima- 
ción al uso del coeficiente alfa de Cronbach. Revista Colombiana de Psiquiatría, 34(4), 572-580.

Chiavenato, I. (2009). Comportamiento Organizacional, la dinámica del éxito en las organizaciones ( $2^{\mathrm{a}}$ ed.). México: Mc Graw Hill.

Codina, A., \& Rivera, A. (2001). Hacia una instrucción basada en la resolución de problemas: Los términos problema, solución y resolución. En P. Gómez \& L. Rico (Eds.), Iniciación a la investigación en didáctica de la matemática. Homenaje al profesor Mauricio Castro (pp. 125-136). Granada, España: Universidad de Granada. Recuperado de https://www.uv.es

Cohen, W. M., \& Levinthal, D. A. (1990). Absorptive capacity: A new perspective on learning and innovation. Administrative Science Quarterly, 35(1), 128-152. doi: $10.2307 / 2393553$

Cozby, P. (2005). Métodos de Investigación del Comportamiento ( $8^{\mathrm{a}}$ ed.). México: McGraw Hill.

Davis, T. R. V., \& Luthans, F. (1979). Leadership re-examined: A behavioral approach. Academy of Management Review, 4(2), 237-248. doi: 10.2307/257777

D'Zurilla, T., \& Chang, E. (1995). The relations between social problem solving and coping. Cognitive Therapy \& Research, 19(5), 547-562. doi: 10.1007/ BF02230513

Echavarri, M., Godoy, J. C., \& Olaz, F. (2007). Diferencias de género en habilidades cognitivas y rendimiento académico en estudiantes universitarios. Universitas Psychologica, 6(2), 319-329. Recuperado de https:// revistas.javeriana.edu.co/index.php/index

Escobar-Pérez, J., \& Cuervo-Martínez, A. (2008). Validez de contenido y juicio de expertos: Una aproximación a su utilización. Avances en Medición 6, 27-36. Recuperado de http://www.humanas.unal.edu.co/psicometria/revista

González, J., Wagenaar, R., \& Beneitone, P. (2004). Tunning-América Latina: Un proyecto de las universidades. Revista Iberoamericana de Educación, 35, 151-164. Recuperado de https://rieoei.org/RIE/index Gros-Salvat, B. (1990). La enseñanza de estrategias de re- solución de problemas mal estructurados. Revista de Educación, 292, 415-433. Recuperado de http:// www.educacionyfp.gob.es/revista-de-educacion/inicio.html

Hemphill, S. A., \& Littlefield, L. (2001). Evaluation of a short-term group therapy program for children with behavior problems and their parents. Behaviour Research and Therapy, 39(7), 823-841. doi: 10.1016/ s0005-7967(00)00058-9

Heppner, P. P., \& Petersen, C. H. (1982). The development and implications of a personal problema-solvin inventory. Journal of Counselling Psychology, 29(1), 66-75. doi: 10.1037/0022-0167.29.1.66

Hernández, R. (2018). Diseño de una escala para evaluar habilidades directivas: Solución de problemas (Tesis de maestría). Universidad Nacional Autónoma de México, México.

Hernández-Sampieri, R., Fernández-Collado, C., \& Baptista-Lucio, P. (2014). Metodología de la Investigación (6 $6^{\mathrm{a}}$ ed.). México, D. F.: Mc Graw Hill.

Huber, G. P. (1992). Toma de decisiones en la gerencia. México: Trillas.

IBM Corp. (2013). IBM SPSS Statistics for Windows, Version 22.0. [software de cómputo]. IBM Corp., Armonk, NY.

Katz, R. (1974). Skills of an effective administrator. Harvard Business Review, 33(1), 33-42. Recuperado de https://hbr.org

Katz, E., \& Ziderman, A. (1990). Investment in general training: The role of information and labour mobility. The Economic Journal, 100(403), 1147-1158. doi: $10.2307 / 2233964$

Lee, W., Park, S., \& Choi, E. (2008). Development of a Korean problem-solving process inventory for adults. Journal of Korean Academy of Fundamentals of Nursing, 15(4), 548-557. Recuperado de https://j. kafn.or.kr

Lévy-Mangin, J., Martín-Fuentes, M., \& Román-González, M. (2006). Optimización según estructuras de covarianzas. En J. Lévy-Mangin \& J. Varela-Mallou 
(Eds.), Modelización con estructuras de covarianzas en ciencias sociales (pp. 9-30). España: Netbiblo. doi: 10.4272/84-9745-136-8.ch2

Lloret-Segura, S., Ferreres-Traver, A., Hernández-Baeza, A., \& Tomás-Marco, I. (2014). El análisis factorial exploratorio de los ítems: Una guía práctica, revisada y actualizada. Anales de Psicología, 30(3), 11511169. Recuperado de https://revistas.um.es/analesps Lorenzo-Seva, U. \& Ferrando, P. (2019) Factor Analysis, Versión 10.10.01 64 bits. [software de cómputo]. Universidad Rovira y Virgili

Łyp-Wrońska, K. (2016). World Class Manufacturing Methodology as an example of problems solution in Quality Management System. Key Engineering Materials, 682, 342-349. doi: 10.4028/www.scientific. net/KEM.682.342

Merrill, K. L., Smith, S. W., Cumming, M. M., \& Daunic, A. P. (2017). A review of social problem-solving interventions: Past findings, current status, and future directions. Review of Educational Research, 87(1), 71-102. doi: 10.3102/0034654316652943

Mumford, M., Marks, M., Zaccaro, S., \& Reiter-Palmon, R. (2000). Development of leadership skills: Experience and timing. Psychology Faculty Publications, 11(1), 87-114. Recuperado de http://digitalcommons. unomaha.edu/psychfacpub/64

Mumford, M. D., Zaccaro, S. J., Harding, F. D., Jacobs, T. O., \& Fleishman, E. A. (2000). Leadership skills for a changing world: Solving complex social problems. The Leadership Quarterly, 11(1), 11-35. doi: 10.1016/S1048-9843(99)00041-7

Nezu, A. M., Nezu, C. M., \& D`Zurilla, T, J. (2014). Terapia de Solución de Problemas: Manual de tratamiento. Bilbao, España: Desclée de Brouwer.

Núñez-López, S., Ávila-Palet, J. E., \& Olivares-Olivares, S. L. (2016). El desarrollo del pensamiento crítico en estudiantes universitarios por medio del aprendizaje basado en problemas. Revista Iberoamericana de Educación Superior, 8(23), 84-103. Recuperado de https://www.ries.universia.unam.mx/index.php/ries

Palomar-Lever, J., Harari-Quinn, A. P., \& Juárez, M. del P.
(2010). Elaboración de una escala de resolución de problemas para adultos (ERPA). Enseñanza e Investigación en Psicología, 15(1), 31-39. Recuperado de https://www.revistacneip.org/index.php/cneip

Patrón-Cortés, R., \& Barroso-Tanoira, F. (2015). Competencias directivas de gerentes de restaurantes turísticos. Un estudio en la ciudad de San Francisco de Campeche, México. Estudios y Perspectivas en Turismo, 24(1), 96-114. Recuperado de https://www. estudiosenturismo.com.ar

Patrón-Cortés, R. M., Pérez-Canul, C. A., \& Medina-Blum, F. (2014). Las competencias directivas más valoradas por las Pymes al elegir a un profesional: Caso tiendas de autoservicio. Revista Iberoamericana de Producción Académica y Gestión Educativa, 1(12). Recuperado de https://www.pag.org.mx/index.php/ PAG/index

Patrón-Cortés, R., Pérez-Canul, C., \& González-García, E. (2015). Competencias directivas más valoradas por el sector turístico al elegir a sus gerentes de hoteles en el sureste de México. Revista Iberoamericana para la Investigación y el Desarrollo Educativo, 2(3). Recuperado de https://www.pag.org.mx/index. php/PAG/index

Pereda-Pérez, F., López-Guzmán-Guzmán, T., \& González-Santa-Cruz, F. (2014). Las habilidades directivas como ventaja competitiva. El caso del sector público de la provincia de Córdoba (España). Intangible Capital, 10(3), 528-561. doi: 10.3926/ic.511

Pérez-Rave, J., \& González-Echavarría, F. (2018). El síndrome de "echar la culpa" desde una mirada sistémica y sus repercusiones para la solución de problemas en la empresa. Revista Ingenierías Universidad de Medellín, 17(33), 187-205. doi: 10.22395/rium. v17n33a10

Quero-Virla, M. (2010). Confiabilidad y coeficiente alpha de Cronbach. Telos, 12(2), 248-252. Recuperado de http://www.redalyc.org/articulo.oa?id=99315569010

Rangel-Carreño, T., Lugo-Garzón, I., \& Calderón, M. (2018). Revisión bibliográfica equipos de trabajo: Enfoque cuantitativo, características e identificación 
de variables que afectan la eficiencia. Ingeniería Solidaria, 14(24) . Recuperado de https://revistas.ucc. edu.co/index.php/in

Razali, N., \& Wah, Y. (2011). Power comparisons of Shapiro-Wilk, Kolmogorov- Smirnov, Lilliefors and Anderson-Darling tests. Journal of Statistical Modeling and Analytics, 2(1), 21-33. Recuperado de http://www.de.ufpb.br/ ulisses/disciplinas/normality_tests_comparison.pdf

Rico, R., Alcover de la Hera, C., \& Tabernero, C. (2010). Efectividad de los equipos de trabajo: Una revisión de la última década de investigación (1999-2009). Revista de Psicología del Trabajo y de las Organizaciones, 26(1), 47-71. Recuperado de http:// scielo.isciii.es/scielo.php?script=sci_arttext\&pi$\mathrm{d}=\mathrm{S} 1576-59622010000100004 \& \operatorname{lng}=\mathrm{es} \& \mathrm{t} \operatorname{lng}=\mathrm{pt}$

Robbins, P., \& Judge, T. (2009). Comportamiento Organizacional (13 ${ }^{\mathrm{a}}$. ed.). México: Pearson/Prentice Hall.

Rodríguez, E., Rabazo, A., \& Naranjo, D. (2015). Evidencia empírica de la adquisición de la competencia de resolución de problemas. Perfiles Educativos, 37(147), 50-66. Recuperado de https:/www.iisue.unam.mx/ perfiles

Ruíz-Méndez, D., \& Vega-Valero, C. (2017). Capacitación basada en evidencias: Una aportación tecnológica desde la psicología. Revista Iberoamericana de Psicología, 10(2), 35-42. doi: 10.33881/2027-1786. rip. 10204

Ruiz, D., Nava, C., \& Carbajal, R. (2016). Issues in organizational assessment: The case of frustration tolerance measurement in Mexico. International Scholarly and Scientific Research \& Innovation, 10(3), 912-916. Recuperado de https://waset.org

San Román-Losada, I. (2014). Factores de comportamiento directivo y su impacto en la calidad. Uruguay: Académica Española.

Sánchez, J., Aguirre, M., Barrales, A., Vergara, J., \& Mota, I. (2015). Las habilidades directivas. En Red Mexicana de Investigadores en Estudios Organizacionales (Ed.), El análisis organizacional en México y América Latina, Retos y Perspectivas a 20 años de Es- tudios (pp. 125-164). México: Hess. Recuperado de http://remineo.org/images/documentos/memorias/ ciao/xiiiciao/aomal.pdf

Shipper, F., \& Davy, J. (2002). A model and investigation of managerial skills, employees' attitudes, and managerial performance. The Leadership Quarterly, 13(2), 95-120. doi: 10.1016/S1048-9843(02)00098-X

Streiner, D. (2003). Being inconsistent about consistency: When coefficient alpha does and doesn't matter. Journal of Personality Assessment, 80(3), 217-222. doi: 10.1207/S15327752JPA8003_01

Tenhula, W., Nezu, A., Nezu, C., Stewart, M., Miller, S., Steele, J., \& Karlin, B. (2014). Moving forward: A problem-solving training program to foster veteran resilience. Professional Psychology: Research and Practice, 45(6), 416-424. doi: 10.1037/a0037150

Timmerman, M. E., \& Lorenzo-Seva, U. (2011). Dimensionality assessment of ordered polytomous items with parallel analysis. Psychological Methods, 16(2), 209-220. doi: 10.1037/a0023353

Whetten, D., \& Cameron, K. (2011). Desarrollo de habilidades directivas ( $8^{\mathrm{a}}$ ed.) México: Pearson Educación.

Yukl, G. (1989). Managerial leadership: A review of theory and research. Journal of Management, 15(2), 251289. doi: 10.1177/014920638901500207 


\section{Apéndice 1}

Escala de solución de problemas para directivos (ESOP-D)

Escala "Solución de problemas"

Instrucciones: Indique con qué frecuencia realiza las siguientes acciones cada vez que se presenta un problema en la organización que depende de usted para ser solucionado.

Marque con una X la opción que más se ajuste a su respuesta. Las opciones son:

\begin{tabular}{ccccc}
\hline Nunca & Casi nunca & Algunas veces & Casi siempre & Siempre \\
\hline 1 & 2 & 3 & 4 & 5 \\
\hline
\end{tabular}

1 Además de mí, otros compañeros de trabajo pueden verificar $\quad \begin{array}{llllll}1 & 2 & 3 & 4 & 5\end{array}$ que el problema se ha resuelto.

2 Busco posibles soluciones a un problema laboral desde $\quad \begin{array}{lllllll}2 & 2 & 3 & 4 & 5\end{array}$ distintos puntos de vista.

$\begin{array}{llllllllll}3 & \text { Realizo un registro de todas las acciones que se llevaron a } & & 1 & 2 & 3 & 4 & 5\end{array}$ cabo en la solución del problema.

$\begin{array}{llllllll}4 & \text { Determino la viabilidad de las alternativas para solucionar el } & & 1 & 2 & 3 & 4 & 5\end{array}$ problema organizacional.

5
Para elegir una solución considero las posibilidades de éxito $\quad \begin{array}{lllll}1 & 2 & 3 & 4 & 5\end{array}$ de cada alternativa.

6
Analizo los factores externos que influyen en el problema. $\quad \begin{array}{lllllll}1 & 2 & 3 & 4 & 5\end{array}$

$\begin{array}{lllllll}7 & \text { Evalúo si la estrategia de solución se ejecutó según lo } & & 1 & 2 & 3 & 4\end{array}$ planeado.

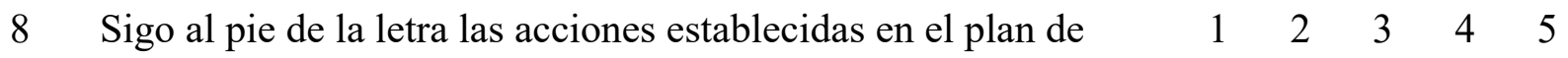
acción para solucionar un problema en mi trabajo.

9 Establezco un tiempo determinado para solucionar el $\quad \begin{array}{llllll}2 & 2 & 3 & 4 & 5\end{array}$ problema.

10 Integro estrategias de solución cuando son compatibles. $\quad \begin{array}{llllll}1 & 2 & 3 & 4 & 5\end{array}$

11 Delimito la situación problemática. $\quad 1 \quad \begin{array}{lllll}2 & 3 & 4 & 5\end{array}$

12 Cuando tomo decisiones en mi puesto de trabajo, intento $\quad \begin{array}{lllllll}1 & 2 & 3 & 4 & 5\end{array}$ predecir los pros y contras de cada alternativa de solución.

13 Encuentro alternativas variadas. $\quad \begin{array}{llllll} & 2 & 3 & 4 & 5\end{array}$

14 Puedo reconocer cuando se solucionó el problema en mi $\quad \begin{array}{llllll}1 & 2 & 3 & 4 & 5\end{array}$ trabajo.

15 Corrijo fallos durante la ejecución del plan de acción para $\quad \begin{array}{lllll}1 & 2 & 3 & 4 & 5\end{array}$ solucionar un problema en mi trabajo.

16 Reúno toda la información posible para delimitar el $\quad \begin{array}{lllll}2 & 3 & 4 & 5\end{array}$ problema. 
17 Cuando me enfrento a un problema conocido identifico las alternativas que me han funcionado.

18 Analizo la relación entre los elementos del problema.

19 Busco información en mi organización acerca del problema.

20 Me anticipo a posibles eventualidades que puede generar la solución del problema.

21 Superviso la ejecución de la alternativa de solución.

22 Trato de anticiparme al resultado que obtendría con cada alternativa de solución.

23 Evalúo si el método elegido es adecuado para resolver el problema en la organización.

24 Elijo la mejor alternativa de solución basándome en los posibles resultados para la organización.

25 Después de llevar a cabo una solución, analizo hasta qué punto el problema organizacional ha mejorado.

26 Comparo las fortalezas y debilidades de cada posible solución antes de tomar una decisión.

27 Verifico la mejora del problema durante la implementación de la solución.

28 Comparo si los resultados organizacionales obtenidos coinciden con los resultados esperados.

29 Utilizo un método para comparar alternativas de solución.

30 Elaboro un plan de acción para resolver el problema.

31 Considero la primera idea de solución que se me ocurre como la mejor alternativa.

32 Me aseguro de que todos los involucrados en el problema participen en la solución.

33 Pienso en la mayor cantidad de posibles soluciones.

34 Establezco metas claras que me permitan identificar cuando ya se resolvió la problemática organizacional.

35 Establezco indicadores para saber en qué momento se resolvió el problema.

36 Verifico si entendí el problema de la organización.

37 Cuando fracaso al solucionar un problema, examino qué fue lo que no funcionó.

38 Evalúo la alternativa que implique el menor gasto de recursos y el mayor beneficio para la organización.

39 Evalúo diversas alternativas para ejecutar el plan de acción.

40 Cuando tengo más de una alternativa de solución elijo la que en mi consideración es la que tiene más probabilidad de solucionar el problema.

$\begin{array}{lllll}1 & 2 & 3 & 4 & 5\end{array}$

$\begin{array}{lllll}1 & 2 & 3 & 4 & 5 \\ 1 & 2 & 3 & 4 & 5 \\ 1 & 2 & 3 & 4 & 5\end{array}$

$\begin{array}{lllll}1 & 2 & 3 & 4 & 5\end{array}$

$\begin{array}{lllll}1 & 2 & 3 & 4 & 5\end{array}$

$\begin{array}{lllll}1 & 2 & 3 & 4 & 5\end{array}$

$\begin{array}{lllll}1 & 2 & 3 & 4 & 5\end{array}$

$\begin{array}{lllll}1 & 2 & 3 & 4 & 5\end{array}$

$\begin{array}{lllll}1 & 2 & 3 & 4 & 5\end{array}$

$\begin{array}{lllll}1 & 2 & 3 & 4 & 5\end{array}$

$\begin{array}{lllll}1 & 2 & 3 & 4 & 5\end{array}$

$\begin{array}{lllll}1 & 2 & 3 & 4 & 5\end{array}$

$\begin{array}{lllll}1 & 2 & 3 & 4 & 5\end{array}$

$\begin{array}{lllll}1 & 2 & 3 & 4 & 5\end{array}$

$\begin{array}{lllll}1 & 2 & 3 & 4 & 5\end{array}$

$\begin{array}{lllll}1 & 2 & 3 & 4 & 5\end{array}$

$\begin{array}{lllll}1 & 2 & 3 & 4 & 5\end{array}$

$\begin{array}{lllll}1 & 2 & 3 & 4 & 5\end{array}$

$\begin{array}{lllll}1 & 2 & 3 & 4 & 5\end{array}$

$\begin{array}{lllll}1 & 2 & 3 & 4 & 5\end{array}$

$\begin{array}{lllll}1 & 2 & 3 & 4 & 5\end{array}$

$\begin{array}{lllll}1 & 2 & 3 & 4 & 5\end{array}$

$\begin{array}{lllll}1 & 2 & 3 & 4 & 5\end{array}$ 